Service social

\title{
La participation sociale des aînés ayant des incapacités : un photoroman pour passer de la marge à l'inclusion
}

\section{Émilie Raymond et Amanda Grenier}

Volume 62, numéro 2, 2016

URI : https://id.erudit.org/iderudit/1038581ar

DOI : https://doi.org/10.7202/1038581ar

Aller au sommaire du numéro

\section{Éditeur(s)}

École de travail social et de criminologie de l’Université Laval

ISSN

1708-1734 (numérique)

Découvrir la revue

Citer cet article

Raymond, É. \& Grenier, A. (2016). La participation sociale des aînés ayant des incapacités : un photoroman pour passer de la marge à l'inclusion. Service social, 62(2), 131-149. https://doi.org/10.7202/1038581ar
Résumé de l'article

Dans le contexte du vieillissement démographique, les discours visant la participation sociale des aînés abondent. Ils s'accompagnent de normes quant au bien-vieillir qui profilent des aînés " actifs ", en « bonne santé ", créant une catégorie distincte, à la marge, pour les personnes ayant des incapacités. Des études montrent d'ailleurs que les aînés ayant des incapacités sont limités dans leur accès aux milieux participatifs. Il s'avère donc crucial de revisiter le carrefour du vieillissement et des incapacités afin de sortir d'une logique de vulnérabilité et de travailler à soutenir la participation sociale de l'ensemble des aînés. L'article présente les résultats d'une recherche-action participative menée pendant trois ans avec des aînés ayant des incapacités physiques. Au cours de ce processus, douze personnes ont créé un photoroman racontant leurs histoires de participation sociale où images et mots défient les stéréotypes quant aux réalités et aux possibilités de participation pour les aînés ayant des incapacités. Deux grands constats émergent. Tout d'abord, une participation sociale soutenue, diversifiée, modulée aux conditions de santé, est souhaitée et expérimentée par les participants. Ensuite, de nombreux obstacles, d'ordre symbolique et matériel, se dressent devant les aînés ayant des incapacités désireux d'intégrer les milieux participatifs fréquentés par les autres aînés. Dans la perspective d'une pratique critique en service social, ces résultats indiquent que, tout en maintenant une approche visant à promouvoir le pouvoir d'agir individuel des aînés ayant des incapacités, il est aussi nécessaire d'adopter une intervention plus collective ou structurelle, afin que les espaces de participation sociale deviennent plus inclusifs et encouragent la participation de l'ensemble des aînés.
Ce document est protégé par la loi sur le droit d'auteur. L'utilisation des services d’Érudit (y compris la reproduction) est assujettie à sa politique d'utilisation que vous pouvez consulter en ligne.

https://apropos.erudit.org/fr/usagers/politique-dutilisation/ 


\title{
La participation sociale des aînés ayant des incapacités : un photoroman pour passer de la marge à l'inclusion
}

\author{
RAYMOND, Émilie \\ Professeure adjointe \\ École de service social \\ Université Laval \\ GRENIER, Amanda \\ Professeure associée \\ Health, Aging and Society \\ McMaster University
}

\section{RÉSUMÉ}

Dans le contexte du vieillissement démographique, les discours visant la participation sociale des aînés abondent. Ils s'accompagnent de normes quant au bien-vieillir qui profilent des aînés « actifs », en «bonne santé », créant une catégorie distincte, à la marge, pour les personnes ayant des incapacités. Des études montrent d'ailleurs que les aînés ayant des incapacités sont limités dans leur accès aux milieux participatifs. II s'avère donc crucial de revisiter le carrefour du vieillissement et des incapacités afin de sortir d'une logique de vulnérabilité et de travailler à soutenir la participation sociale de l'ensemble des aînés. L'article présente les résultats d'une recherche-action participative menée pendant trois ans avec des aînés ayant des incapacités physiques. Au cours de ce processus, douze personnes ont créé un photoroman racontant leurs histoires de participation sociale où images et mots défient les stéréotypes quant aux réalités et aux possibilités de participation pour les aînés ayant des incapacités. Deux grands constats émergent. Tout d'abord, une participation sociale soutenue, diversifiée, modulée aux conditions de santé, est souhaitée et expérimentée par les participants. Ensuite, de nombreux obstacles, d'ordre symbolique et matériel, se dressent devant les aînés ayant des incapacités désireux d'intégrer les milieux participatifs fréquentés par les autres aînés. Dans la perspective d'une pratique critique en service social, ces résultats indiquent que, tout en maintenant une approche visant à promouvoir le pouvoir d'agir individuel des aînés ayant des incapacités, il est aussi nécessaire d'adopter une intervention plus collective ou structurelle, afin que les espaces de participation sociale deviennent plus inclusifs et encouragent la participation de l'ensemble des aînés. 


\section{INTRODUCTION}

Depuis le début des années 2000, les discours sur la participation sociale des aînés dans le contexte du vieillissement démographique abondent, au Québec (Gouvernement du Québec, 2007, 2009b, 2012a) comme sur la scène internationale (Organisation des Nations unies, 2002, 2008; Organisation mondiale de la santé, 2002, 2007). Perçu à la fois comme l'occasion de revisiter notre rapport collectif au grand âge et comme une partie de la solution pour faire face au fardeau économique attribué au vieillissement démographique (United Nations Organization, 2005), l'appel participatif vise un certain type d'audience et un certain type de participation. Au Québec, les aînés « actifs », en « bonne santé », sont les premiers ciblés, et on attend d'eux qu'ils maintiennent des activités contributives, travail salarié, bénévolat ou proche aidance (Gouvernement du Québec, 2012b). D'ailleurs, dans la première politique québécoise en matière de vieillissement, la première orientation affirme que « vieillir et vivre ensemble, c'est participer dans sa communauté ", idée maîtresse qui se traduit par l'implication bénévole et le soutien aux travailleurs expérimentés dans les milieux de travail (Gouvernement du Québec, 2012a). Si cette norme participative peut correspondre aux désirs de nombreux aînés (Statistique Canada, 2012, 2009), elle coïncide plus difficilement avec les besoins ou les réalités des personnes ayant des incapacités, notamment parce que divers facteurs freinent leur accès aux milieux de participation s'adressant aux aînés, et également parce que leurs conditions de vie et de santé peuvent contraindre leurs choix en matière de participation (Raymond, Gagné, Sévigny et Tourigny, 2008; Raymond, Sévigny et Tourigny, 2011, 2012). Pourtant, nous pouvons penser que ces personnes, qui ont évolué toute leur vie dans une société souhaitant et aidant leur intégration dans les mondes scolaire et professionnel (Gouvernement du Québec, 2005a, 2009a), ont le droit de continuer à choisir leurs activités et à être des citoyens à part entière durant le vieil âge.

Cet article expose les résultats d'une recherche menée avec l'intention : 1) de donner la parole à des personnes vieillissant avec des incapacités physiques pour qu'elles puissent raconter leurs visions et leurs expériences en matière de participation sociale ${ }^{1}$; et 2) de contester l'image un peu lisse des modèles dominants du vieillissement ${ }^{2}$. Dans le cadre d'une recherche-action participative, 12 aînés ayant des incapacités physiques, auditives ou visuelles ont travaillé pendant trois ans à créer un photoroman disant en mots et en images leur rapport à la participation sociale. Ce travail visait à répondre aux questions suivantes: a) comment des personnes aînées ayant des incapacités conçoivent-elles leur participation à la société ? b) quelles pratiques développent-elles pour vivre cette participation $^{3}$ ? Dans le cadre du présent texte, nous répondrons à ces questions en mettant nos résultats en rapport avec l'intervention en service social. Nous nous inspirerons d'un article de Paul Stepney (2006) pour montrer que le Projet Photoroman illustre bien une pratique critique de cette discipline. Dans les deux cas, l'attention portée au contexte des personnes et à leur pouvoir d'agir s'inscrit dans un projet émancipatoire. Ainsi, la manière dont les scénarios du photoroman ont été

\footnotetext{
${ }^{1}$ Le présent article ne fournira pas un cadre conceptuel de participation sociale, puisque la recherche dont il est tiré utilisait une approche inductive pour construire avec les participants leurs visions et leurs pratiques de la participation.

${ }^{2}$ L'approche inductive présentée dans la note précédente s'oppose en quelque sorte à la définition donnée à la participation sociale par les politiques sociales, définition dont sont déduits les comportements participatifs reconnus (travail salarié, bénévolat et proche aidance, tel qu'énoncés précédemment).

3 Une troisième question concernait le rapport entre ces conceptions et pratiques de la participation et les politiques québécoises du vieillissement, mais elle ne sera pas abordée ici.
} 
élaborés coïncide avec la technique de l'incident critique proposée par Stepney, comme un processus pour percevoir, revisiter et transformer les discours et les structures générateurs d'inégalités sociales.

La prochaine section situera le thème de la participation sociale des aînés dans le contexte où se croisent les politiques de nouvelle gestion publique et le vieillissement démographique. Nous nous intéresserons ensuite à l'attention limitée accordée jusqu'ici aux aînés ayant des incapacités dans les écrits scientifiques et la «littérature grise », éclairant ainsi une marginalisation faisant écho à la difficulté, pour les personnes concernées, de concrétiser leurs projets de vie dans la vieillesse. Les principes d'une pratique critique du service social seront ensuite définis dans le cadre théorique, avant d'expliquer la méthode de la recherche-action participative réalisée. Suivront deux grands constats relativement aux résultats, chacun appuyé par un récit du photoroman et montrant, premièrement, que les participants de la recherche souhaitent à la fois poursuivre et réinventer leur participation sociale et, deuxièmement, qu'il ne suffit pas de vouloir pour pouvoir participer en raison des différents obstacles limitant l'accès aux possibilités de participation sociale. La discussion des résultats utilisera la technique de l'incident critique (Stepney, 2006) pour mettre en relief la manière dont le Projet Photoroman a d'abord permis d'identifier, d'analyser et de déconstruire des normes participatives incapacitantes, pour ensuite réélaborer un discours plus nuancé et inclusif.

\section{RECENSION DES ÉCRITS : APPARENCES ET ABSENCES DES DISCOURS PARTICIPATIFS}

\section{La participation sociale des aînés à l'aune de la nouvelle gestion publique}

Depuis le début des années 2000, le concept de participation sociale des aînés est omniprésent dans les discours publics sur le vieillissement. Il était apparu dans les écrits scientifiques en gérontologie une décennie plus tôt, sous les traits d'un élément-clé des modèles de vieillissement productif, réussi et actif (respectivement Morrow-Howell, Hinterlong et Sherraden, 2001; Rowe et Kahn, 1998; Walker, 2002). Du point de vue politique, l'association entre une participation sociale active et une trajectoire optimale de vieillissement a été célébrée lors de la Deuxième Assemblée mondiale sur le vieillissement, tenue à Madrid en 2002. À partir de ce moment, les aînés ont été présentés comme des acteurs sociaux responsables de leur trajectoire de vieillissement, une rupture de ton notoire par rapport aux conclusions de la Première Assemblée mondiale sur le vieillissement (Vienne, 1982), dirigées vers la protection d'aînés perçus comme des êtres vulnérables (Organisation des Nations unies, 1982).

Ce programme participatif peut être vu comme partie prenante d'une réingénierie globale du modèle d'État-providence, approche de renouvellement qui se concentre sur la responsabilité et la participation individuelles pour faire face aux risques et aux problèmes sociaux (Rosanvallon, 2000, 2008). En fait, le concept de participation est au centre des régimes de citoyenneté qui ont gouverné les démocraties capitalistes depuis les années 1990 (Jenson et Saint-Martin, 2003). Dans ces gouvernements, l'assistance publique est organisée et offerte par un quatuor de partenaires : l'État, le marché, le tiers secteur (incluant notamment l'action communautaire autonome, le mouvement coopératif et l'économie sociale), et les individus et leur famille (Evers, Pilj et Ungerson, 1994). Les citoyens sont tenus de devenir des acteurs de ce partenariat; autrement dit, on les convie à faire des choix et à avoir des habitudes de vie favorables à leur bonne santé. En ce sens, la participation sociale des aînés, dont les 
effets positifs sur la santé sont largement étudiés et reconnus (Zedlewski et Butrica, 2007), apparaît comme une condition de leur inclusion ${ }^{4}$ dans la communauté.

Au Québec, c'est en 2004 que le nouveau gouvernement, issu du Parti libéral, a mis en place un grand projet de réingénierie de l'État, avec une stratégie centrale basée sur la participation citoyenne et des partenariats entre les secteurs public et privé et du tiers secteur (Brunelle, Harvey et Bédard, 2005; Côté et Lévesque, 2009). Le vieillissement a été ciblé en tant que priorité d'intervention suite à un Forum des générations, et le ministère de la Famille et des Aînés a été créé dès l'année suivante. Ce nouveau ministère publia rapidement le rapport Une pleine participation des aînés au développement du Québec : Afin de construire un Québec pour tous les âges (Gouvernement du Québec, 2005b). À partir de ce moment, la participation a été considérée comme un objectif dans toutes les politiques québécoises sur le vieillissement. Si cette centralité n'est pas un problème en soi, les normes autour d'un vieillissement optimal différencient les aînés considérés comme " actifs 》 des aînés considérés comme malades ou limités, menant à une prescription participative fort différente selon la catégorie à laquelle on appartient (Raymond et Grenier, 2012, 2013; Raymond, Grenier et Lacroix, 2016). Enracinées dans ces discours, les pratiques professionnelles peuvent devenir marginalisantes, pour les aînés ayant des incapacités, en assignant à ces derniers des possibilités restreintes de participation sociale (Lacroix et Raymond, 2014; Raymond et Pomerleau, 2011).

\section{Vieillissement et incapacités, un carrefour (encore) peu fréquenté}

Depuis quelques années, des chercheurs et des intervenants sociaux ont invité à un rapprochement entre le champ de la gérontologie et celui des incapacités (Bickenbach et al., 2012; Jeppsson Grassman et Whitaker, 2013; National Council on Ageing and Older People et National Disability Authority, 2006). Pendant longtemps, les connaissances et les pratiques liées à chaque domaine se sont développées en parallèle, chacun se construisant en rejetant l'étiquette délétère accolée à l'autre : une exclusion mutuelle qui peut également s'observer dans le mouvement associatif de l'un et l'autre champ. Pendant que les organisations de personnes ayant des incapacités peinent à répondre aux besoins de leurs membres plus âgés, qui s'éloignent des sphères habituelles de revendication politique, les organisations d'aînés, pour leur part, tendent à écarter ou à éviter les personnes ayant ou développant des incapacités, notamment parce que leur image dérange et qu'on voit leurs demandes d'accommodements comme affectant le cours et le rythme « normaux » des activités (Bigby et Balandin, 2005; De Buys, Boulton-Lewis et Tedman-Jones, 2008 ; Raymond, Grenier et Hanley, 2014; Savard, Leduc, Lebel, Béland et Bergman, 2009).

Du côté de la gérontologie, les modèles contemporains (Morrow-Howell, Hinterlong et Sherraden, 2001; Rowe et Kahn, 1998) ont, depuis la fin des années 1990, traité les incapacités comme une anomalie déviant du vieillissement optimal. Deux cheminements sont en quelque sorte dichotomisés, opposant un mode de vie actif combinant loisirs et implication sociale, et une trajectoire marquée par les limitations, la dégénérescence (Biggs, 2001, 2004; Katz, 2005; Laliberté Rudman, 2006, 2011). Bien que le risque de vivre avec des incapacités augmente avec l'âge et que 26,1 \% des personnes de 65

\footnotetext{
${ }^{4}$ L'inclusion sociale consiste à faire en sorte que tous les enfants et adultes aient les moyens de participer et de contribuer à leur communauté et à la société. Cinq fondements la caractérisent : la reconnaissance et la valorisation à la fois des individus et du bien commun; l'accès à des occasions de développement humain; la possibilité et la capacité d'être impliqué dans les prises de décisions qui les concernent ; la proximité et le partage d'espaces physiques et sociaux facilitant les interactions; le bien-être matériel (Laidlaw Foundation, www.laidlawfdn.org).
} 
ans et plus aient une incapacité modérée ou sévère (Gouvernement du Québec, 2013), cette situation est encore traitée comme une exception. Dans plusieurs documents gouvernementaux du Québec, on affirme que la majorité des aînés sont « en bonne santé » et « actifs », et que seule une minorité d'entre eux a besoin de soutien dans la réalisation de ses activités quotidiennes (Gouvernement du Québec, 2005b, 2007, 2009c, 2012a). Les aînés ayant des incapacités sont donc considérés sous l'angle d'un groupe à risque dont les besoins sont médicalisés et qui reste de facto exclu des espaces participatifs (Laliberté Rudman, Huot, Klinger, Leipert et Spafford, 2010; Minkler et Fadem, 2002; Raymond et Grenier, 2013).

Quant au champ du handicap, il continue de défendre une vie pleine, satisfaisante, pour les personnes ayant des incapacités, à toutes les «grandes étapes » de la vie, comme la scolarisation, la parentalité et l'emploi (Ingvaldsen et Balandin, 2011; Priestley, 2003). Le groupe des aînés reste toutefois à la marge de ces discours, comme en témoigne le fait qu'aucune des 24 priorités d'Enable, l'agence des Nations unies consacrée aux personnes ayant des incapacités, ne concerne le vieillissement (United Nations Organization, 2014). II semble qu'il puisse s'agir d'une forme d'âgisme amenant à confondre vieillissement et perte de capacités, une association heurtant les efforts du mouvement des personnes ayant des incapacités pour se dissocier d'images évoquant la dépendance et le déclin (Jönson et Taghizadeh Larsson, 2009). Par ailleurs, lorsqu'elles s'intéressent à la participation sociale des aînés ayant des incapacités, les études sur le handicap tendent à mettre l'accent sur les activités semblables au travail salarié (work-liked activities) comme le bénévolat, tandis que les activités culturelles, politiques et de loisir sont l'objet d'une attention moindre (Balandin, Llewelly, Dew et Ballin, 2006; Narushima, 2005; Tang, 2009).

Au demeurant, la possibilité pour les aînés ayant des incapacités d'avoir une participation sociale correspondant à leurs préférences et à leurs réalités semble modulée par diverses sources de représentation qui associent la participation à une performance contributoire, individualisent les parcours de participation et différencient les aînés en bonne santé des « autres 》 (Raymond et Grenier, 2013). Il est donc important d'analyser et d'investir la thématique à partir d'une perspective critique apte à rendre compte des impasses de la trame participative des discours sur le vieillissement, et surtout à proposer des changements favorisant le caractère inclusif des milieux de participation sociale.

\section{CADRE THÉORIQUE : POUR UNE PRATIQUE CRITIQUE DU SERVICE SOCIAL}

Afin de mettre en rapport le Projet Photoroman et l'intervention en service social, nous utiliserons les repères d'une approche critique du service social, dont l'objectif est de tenir compte des interactions du contexte et de la capacité d'action des individus tout en élaborant des outils pour défier les discours de marginalisation et construire le changement social (Stepney, 2006). Cette approche a été élaborée au Royaume-Uni, dans le contexte politique issu des années 1990 et de la Third Way, menant à une pratique différente du service social, marquée par l'érosion des services publics et un recours plus résolu au tiers-secteur et au marché privé pour diminuer les coûts dans une logique d'efficacité entrepreneuriale. S'en est suivie une accentuation du rôle normalisateur de la profession, d'abord ancrée dans la gestion des groupes à risque, et donc un affaiblissement de la capacité des travailleurs sociaux à développer, à long terme, des initiatives progressistes, combinant protection des personnes en situation de vulnérabilité et transformation sociale. Lorsque Stepney analyse les effets pervers d'une politique d'intervention précoce auprès des enfants dont les cinq mots d'ordre sont "être en santé », « rester en sécurité », « profiter de la vie et se réaliser », « contribuer positivement à la communauté », 
et "atteindre un bien-être économique ", nous ne pouvons que retrouver l'esprit des politiques québécoises du vieillissement à l'égard d'une participation sociale des aînés, ancré dans une rhétorique de contribution socioéconomique, de responsabilisation individuelle et de polarisation de la "vie active » et de la «perte d'autonomie » (Raymond et Grenier, 2012, 2013).

Afin de permettre aux travailleurs sociaux de renouer avec une pratique plus progressiste, déjouant l'enfermement dans l'individualisation des problèmes sociaux et l'activation des populations considérées vulnérables, trois pistes d'action sont proposées par Stepney : 1) l'établissement de nouvelles collaborations (entre les professions du social et de la santé et avec les usagers des services); 2) le travail dans la communauté (pour reconstruire à la fois l'analyse des problèmes sociaux et les mesures locales et globales pour les résoudre); et 3 ) le recours aux résultats de la recherche-action permettant un croisement des savoirs scientifiques et des savoirs d'expériences (nourrissant des interventions adaptées aux réalités et au développement du pouvoir d'agir des personnes principalement concernées par les problèmes ciblés). Dans le cadre du Projet Photoroman, ces principes peuvent aider non seulement à comprendre notre processus de recherche, mais également les résultats obtenus.

En effet, sur le plan du travail de terrain, l'article de Stepney propose une pratique schématique basée sur la technique de l'incident critique, qui consiste en quatre étapes : 1) identifier un incident critique et détailler ce qui s'est passé (un incident critique correspond à un moment durant lequel l'intervenant doit suspendre son intervention parce que quelque chose a presque mal tourné ou dérapé); 2) analyser l'incident en fonction du contexte théorique et politique, en utilisant une perspective structurelle visant à éclairer les discours dominants et les réponses à l'incident données par les différents acteurs impliqués; 3) déconstruire l'argumentaire oppressif utilisé pour soutenir les discours dominants; puis explorer de quelle manière cet argumentaire pourrait être modifié en employant des cadres et discours suggérant des réalités alternatives; 4) reconstruire et recréer des actions et des processus émancipatoires. Dans le cas qui nous occupe, la discussion des résultats montrera que le Projet Photoroman a permis d'opérer cette critique de discours producteurs de marginalisation et leur réinvention en vue de rapports sociaux inclusifs par les personnes principalement concernées ellesmêmes, soit les aînés ayant des incapacités.

\section{MÉTHODE : UNE RECHERCHE-ACTION PARTICIPATIVE POUR RÉINVENTER LES DISCOURS}

Les résultats exposés dans l'article sont issus du Projet Photoroman, une recherche-action participative qui s'est déroulée pendant trois ans (de mai 2011 à juin 2014) dans un organisme d'action communautaire autonome de Québec : le Carrefour familial des personnes handicapées. Le projet faisait suite à des réflexions entreprises par les membres de l'organisation depuis quelques années sur la manière d'adapter leurs réflexions et leurs services aux réalités des membres vieillissants. Suite à un forum communautaire organisé par le Carrefour à l'automne 2010 pour parler des réalités du vieillissement quand on a des incapacités (Carrefour familial des personnes handicapées du Québec, 2011; Raymond et Pomerleau, 2011), cette organisation et deux autres, à savoir l'Association des personnes ayant une déficience de l'audition et le Regroupement des personnes handicapées visuelles 03-12, ont décidé de s'unir et de réaliser un projet visant à passer de la parole aux actes pour soutenir la pleine participation sociale de leurs membres âgés. Après la diffusion de l'information concernant ce projet auprès des membres des trois organismes, 12 aînés ont souhaité faire partie de la démarche. 
Le projet visait deux objectifs principaux : offrir aux participants un espace pour partager leurs histoires, préoccupations et désirs sur le plan de la participation à la société, ainsi que faire entendre cette prise de parole auprès des différents auditoires concernés (pouvoirs publics, mouvements sociaux, réseaux public et communautaires de services). Durant les trois années du projet, la réalisation du photoroman Entre vous et nous ${ }^{5}$ a demandé une trentaine de rencontres de travail et l'apport de différents artistes (photographe, caricaturiste, auteure), en plus du soutien d'une animatrice, de la directrice de Carrefour et d'une bénévole (la première auteure du présent article), à cette époque, étudiante au doctorat.

La méthode de la recherche-action participative a été choisie parce qu'elle mène à travailler en collaboration avec des groupes de personnes touchées par des problèmes afin à la fois de produire des connaissances sur ces problèmes et de développer des solutions (Chevalier et Buckles, 2008; Reason et Bradbury, 2008). Elle demande de construire une relation entre chercheurs et participants permettant un partage des expertises, des pouvoirs et des responsabilités; une telle posture méthodologique est un outil judicieux pour produire des données utiles et rigoureuses, obtenir des bénéfices mutuels et permettre le changement social (Bradbury et Reason, 2003; Fals Borda, 2001; Pain, Kindon et Kesby, 2007). Dans le cas du Projet Photoroman, nous avons généré des connaissances scientifiques inédites sur la participation sociale des aînés ayant des incapacités, tout en renforçant le pouvoir d'agir individuel et collectif des participants. Par exemple, ces derniers se sont (ré)approprié leur trajectoire de participation, ont été reconnus pour leur expertise, sont devenus les auteurs d'un photoroman et ont assumé la fonction de porte-parole au moment de la diffusion du document.

Menée par l'auteure de l'article dans le cadre d'une étude de cas intégrée à la recherche-action participative, la collecte de données a combiné des entretiens individuels, des séances de travail en groupe et des périodes d'observation participante. Premièrement, une entrevue a été réalisée avec chacun des participants au projet (voir Tableau 1) afin d'explorer leurs pratiques de participation sociale, à propos de l'expérience d'une longue vie avec des incapacités et de leurs projets participatifs pour l'avenir. Deuxièmement, les transcriptions de plusieurs réunions de groupe consacrées à la création du photoroman ont été utilisées comme source d'information. Troisièmement, le processus d'écriture collective des scénarios du photoroman et des séances de photographie ont fait l'objet d'une observation participante.

Tableau 1. Caractéristiques des participants de la recherche

\begin{tabular}{|l|l|l|l|l|}
\hline Participants & $\begin{array}{c}\text { Âge } \\
\text { en } \\
\mathbf{2 0 1 2}\end{array}$ & $\begin{array}{c}\text { État civil et } \\
\text { situation } \\
\text { familiale }\end{array}$ & $\begin{array}{c}\text { Expérience à long terme de } \\
\text { travail salarié }\end{array}$ & $\begin{array}{l}\text { Incapacités telles que décrites } \\
\text { aux membres du groupe }\end{array}$ \\
\hline André La. & 67 & Marié, un enfant & Fonctionnaire, retraité & Quasi tétraplégique, de naissance \\
\hline André Le. & 71 & $\begin{array}{l}\text { Veuf, deux } \\
\text { enfants, vit seul }\end{array}$ & Aucun lien d'emploi & $\begin{array}{l}\text { Paraplégique, accident de voiture, } \\
\text { début de l'âge adulte }\end{array}$ \\
\hline Christian & 60 & $\begin{array}{l}\text { Célibataire, vit } \\
\text { seul }\end{array}$ & Fonctionnaire, en emploi & $\begin{array}{l}\text { Tétraplégique, accident de voiture, } \\
\text { début de l'âge adulte }\end{array}$ \\
\hline Claude & 68 & $\begin{array}{l}\text { Divorcé, union } \\
\text { libre, 4 enfants }\end{array}$ & Cadre, retraité & $\begin{array}{l}\text { Paraplégique, accident de voiture, } \\
\text { enfance }\end{array}$ \\
\hline
\end{tabular}

${ }^{5}$ Disponible gratuitement sur le site Internet suivant : http://www.cfph.org/publications.html. 


\begin{tabular}{|c|c|c|c|c|}
\hline Participants & $\begin{array}{l}\text { Âge } \\
\text { en } \\
2012\end{array}$ & $\begin{array}{l}\text { État civil et } \\
\text { situation } \\
\text { familiale }\end{array}$ & $\begin{array}{l}\text { Expérience à long terme de } \\
\text { travail salarié }\end{array}$ & $\begin{array}{l}\text { Incapacités telles que décrites } \\
\text { aux membres du groupe }\end{array}$ \\
\hline Francine & 57 & $\begin{array}{l}\text { Divorcée, } 2 \\
\text { enfants, vit avec } \\
\text { ses parents }\end{array}$ & Représentante, en emploi & $\begin{array}{l}\text { Malentendante, début de l'âge } \\
\text { adulte }\end{array}$ \\
\hline Gilles & 66 & Marié, 1 enfant & Fonctionnaire, retraité & Malentendant, de naissance \\
\hline Lise & 67 & $\begin{array}{l}\text { Divorcée, union } \\
\text { libre, } 2 \text { enfants }\end{array}$ & Infirmière auxiliaire, retraitée & Malentendante, de naissance \\
\hline Muriel & 64 & $\begin{array}{l}\text { Célibataire, vit } \\
\text { seule }\end{array}$ & Aucun lien d'emploi & $\begin{array}{l}\text { Difficulté de mobilité et } \\
\text { fibromyalgie, accident de travail, } \\
\text { début de l'âge adulte }\end{array}$ \\
\hline Normand & 72 & $\begin{array}{l}\text { Divorcé, en union } \\
\text { libre }\end{array}$ & Technicien, retraité & $\begin{array}{l}\text { Handicapé visuel, de naissance } \\
\text { Malentendant, début de la } \\
\text { soixantaine }\end{array}$ \\
\hline Rachel & 61 & $\begin{array}{l}\text { Célibataire, vit } \\
\text { seule }\end{array}$ & Fonctionnaire, retraitée & $\begin{array}{l}\text { Malentendante, âge adulte (dans la } \\
\text { quarantaine) }\end{array}$ \\
\hline Réal & 56 & Veuf, vit seul & $\begin{array}{l}\text { Organisateur } \\
\text { communautaire, en emploi }\end{array}$ & $\begin{array}{l}\text { Handicapé visuel et malentendant, } \\
\text { début de l'âge adulte }\end{array}$ \\
\hline Thérèse & 72 & $\begin{array}{l}\text { Célibataire, vit } \\
\text { seule }\end{array}$ & Fonctionnaire, retraitée & $\begin{array}{l}\text { Paraplégique suite à une paralysie } \\
\text { cérébrale, de naissance }\end{array}$ \\
\hline
\end{tabular}

Les données collectées ont fait l'objet d'une analyse qualitative de contenu consistant en un cheminement en spirale, fait de cercles analytiques itératifs : gestion des données (organisation des données en fonction de fichiers); lecture et rédaction de mémos (réflexion et la codification du matériel); description et interprétation des données (création de catégories de sens et mise en relation avec les différents contextes); représentation et visualisation (création d'outils pour présenter les résultats) (Creswell, 2007; Hammersley et Atkinson, 2007). Nous avons cherché à construire une compréhension en profondeur, contextualisée, de la manière dont les participants interprètent et vivent leur participation sociale au cœur de leurs réalités et arrangements de vie, afin, notamment, de nourrir des interventions en service social plus sensibles à la diversité des vieillissements. La consistance et la fiabilité des résultats ont été rehaussées par trois stratégies principales : engagement prolongé de la chercheure principale sur le terrain de recherche; triangulation des sources de données; validation des données par les participants (Padgett, 2008).

\section{RÉSULTATS : RÉCITS DU POSSIBLE}

En réponse aux questions de recherche concernant les visions et les pratiques des participants concernant leur participation sociale, deux constats principaux émergent des dix récits issus du photoroman Entre vous et nous. Tout d'abord, une participation sociale soutenue, diversifiée, accommodée en fonction des conditions de santé, est souhaitée et expérimentée par les participants. Ensuite, de nombreux obstacles, d'ordre symbolique et matériel, se dressent devant les aînés ayant 
des incapacités désireux d'intégrer les milieux participatifs regroupant des aînés. Nous approfondirons maintenant ces constats à partir de deux scénarios du photoroman 6 .

\section{La participation comme processus pluriel d'inclusion}

Le Photoroman montre ses protagonistes comme des personnes expérimentées, compétentes, conformant une iconographie renouvelée du vieillissement. Les aînés ayant des incapacités sont déplacés depuis les marges jusqu'au cœur d'un discours sur la participation célébrant la diversité. L'histoire racontée dans l'lllustration 1 - celle de deux personnes utilisant à la fois des aides techniques, leur débrouillardise et leur humour pour réaliser des activités de la vie quotidienne - brouille et réinterprète les identités habituellement associées au fait de vieillir avec des incapacités. Ainsi, le scénario présente ses personnages comme des « citoyens ordinaires », organisés, prêts à affronter les défis de la vie quotidienne. Sans être perçus comme vulnérables, ils ne nient pas non plus les difficultés potentielles associées au fait de vivre avec des incapacités.

\section{Illustration 1. Une canne est une canne}
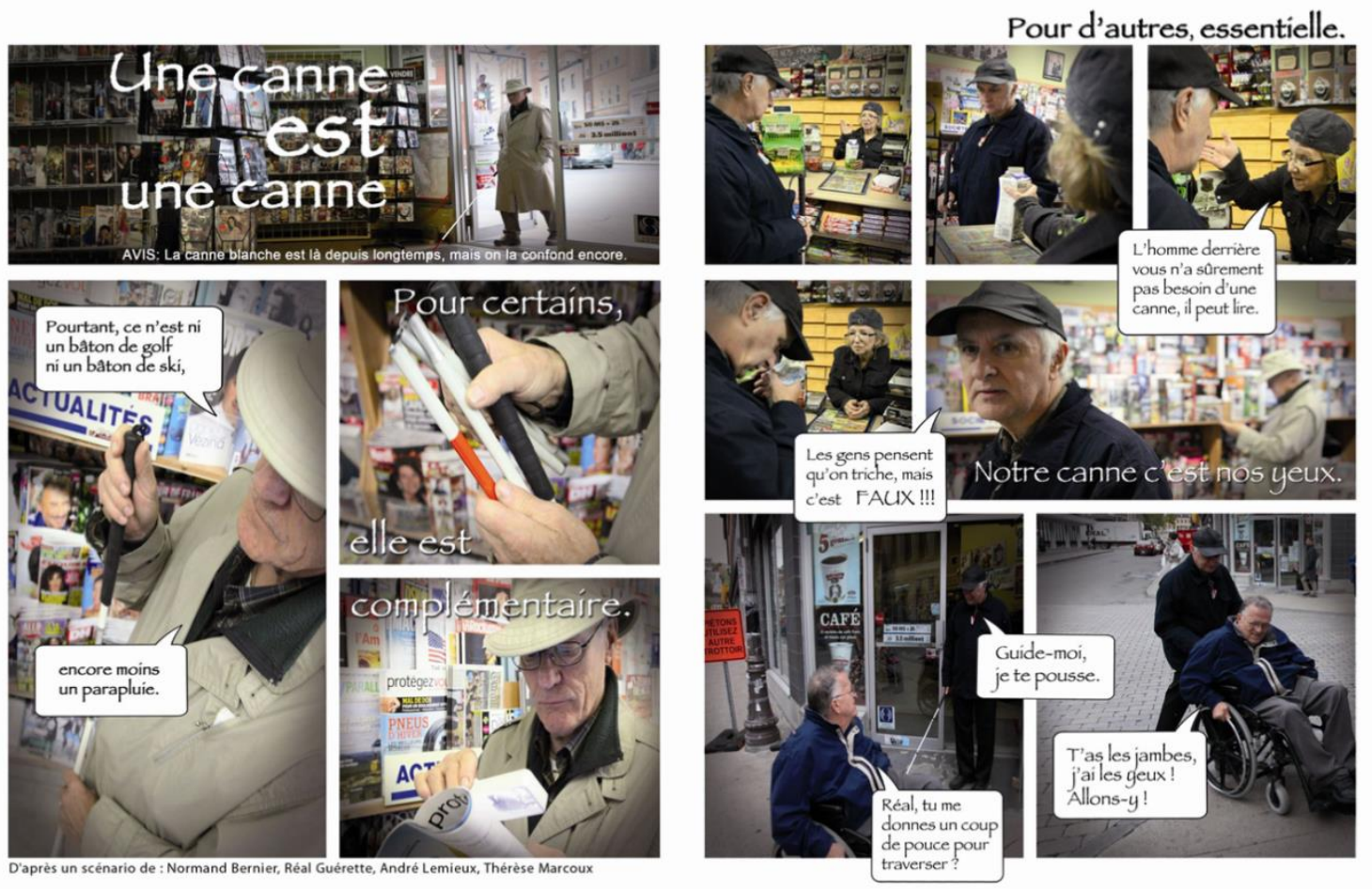

Tous les participants du projet étaient membres d'une organisation de défense des droits des personnes ayant des incapacités. II n'apparaissait donc pas surprenant de les entendre parler de leur détermination à vivre une pleine autodétermination non en tant que héros, mais bien en tant que personnes «comme les autres » aspirant à avoir une « vie normale »:

Ma manière de voir la participation sociale, c'est la participation qui veut dire inclusion. Ça veut

\footnotetext{
${ }^{6}$ Le photoroman est disponible dans son entièreté sur le site Internet suivant <http://www.cfph.org/publications.html>.
} 
dire que comme personne avec des incapacités, tu dois te prendre en main (Christian).

C'est drôle, le mot participation, c'est spécial. Pourquoi tu ne dis pas vivre dans la société comme tout le monde, au lieu de participer? (Muriel)

Nos données montrent que pour les participants, les incapacités sont une partie, un élément parmi d'autres de leur trajectoire de vie. Bien que les stéréotypes au sujet des incapacités dans la vieillesse puissent assigner les personnes à l'inactivité et au désinvestissement, les récits du photoroman montrent que les participants réalisent une pluralité d'activités éducatives, professionnelles, familiales, sociales, culturelles et politiques. Au sujet de cette posture d'acteurs en contrôle de leur existence, un participant exprime l'opinion suivante :

Souvent quand on voit une personne ayant des incapacités qui réussit ou qui fait quelque chose, c'est un surhomme ou un superman ou une superwoman. Des fois, c'est bien normal. Supposons qu'il a réussi à travailler et à vivre une vie de couple, une vie de famille, et à fonctionner dans la vie un peu comme tout le monde, il n'est plus superman ou superwoman pour autant, c'est normal (Réal).

Tout au long du projet et dans le photoroman, les participants ont montré comment leur quotidien est le reflet d'une version personnelle de l'autonomie, fruit de leur longue expérience d'ajustement dans un monde souvent peu sensible à leurs besoins. Pour eux, la participation durant leur vieillisse implique de maintenir leur capacité de décider de quoi sont faits leurs choix d'activité et d'engagement, par le biais de décisions et de stratégies conscientes, réfléchies.

Aider à rester en santé, ça, c'est à nous autres. Bien se nourrir, faire des exercices, ne pas fumer. Ça fait que bien vieillir, on a une part d'action à faire pour que ça aille bien. II ne faut pas toujours profiter de la société (Rachel).

Ma femme et moi, on a vendu notre maison il y a cinq ans. (...) Forcé de vendre ces lieux-là parce qu'on n'avait pu le " guts » de trouver quelqu'un pour faire le gazon, ramasser les feuilles et faire la neige, poser mon maudit garage tempo. (...) Quand tu rentres dans un appartement, tu te rends compte que tes capacités ont diminué (André La).

À ce propos, même si les participants ont développé une expertise sur le plan de l'autodétermination, ils relatent que le fait de vieillir induit de nouvelles exigences pour continuer d'être au centre des choix façonnant leur existence. D'un côté, ils répètent que leur participation ne diminue pas, mais qu'elle évolue en fonction de leurs désirs ou de leur situation. D'un autre côté, ils parlent abondamment du fait que leurs incapacités les font « vieillir plus vite " que les autres aînés, ce qui peut moduler leurs modes participatifs en adoptant des tactiques visant à préserver les capacités restantes.

Le corps est plus sollicité, forcément. L'espérance de vie est moins longue, certain, et pour les capacités physiques, tu en as moins à perdre. (...) C'est comme je dis, on en a moins en banque, donc nos réserves s'épuisent plus rapidement (Claude).

J'aimerais ça aller aux réunions de la municipalité, mais à un moment donné, ça me fatigue, donc je me dis qu'il faut que je garde mes énergies (...) J'aimerais ça m'impliquer là-dedans, mais non, je ne peux pas (Thérèse). 
Ce qu'on peut comprendre de ces témoignages, c'est que les incapacités influent sur le sens de la participation et sur ses pratiques, mais pas toujours de la façon attendue. La participation est inventée et réinventée au gré du temps, à mesure que les personnes continuent de se débrouiller dans un environnement mal adapté, de s'adapter à des conditions de santé changeantes. Sur ce plan, le message central du photoroman est que la participation, lorsqu'elle s'insère correctement dans les préférences et les circonstances d'une personne, lorsqu'elle est soutenue par les accommodements et aides techniques nécessaires, peut lui donner les moyens de préserver ses capacités et sa vitalité grâce à la connexion qu'elle génère avec la communauté, ses membres et ses organisations. Autrement dit, la participation devient vectrice d'inclusion sociale.

\section{La participation comme une lutte à mener}

La plupart des membres du Projet Photoroman souhaitent vivre une vieillesse stimulante, inventive, à l'image des idées promues relativement au vieillissement dit actif. Toutefois, nos données ont permis de documenter de nombreuses situations de disjonction ou de rupture entre les besoins et les désirs des participants quant à leur participation à la société, et leurs possibilités de les réaliser.

Les contraintes à la participation peuvent être d'ordre relationnel. Des participants font référence au fait qu'ils se sentent jugés, discriminés, rejetés dans plusieurs milieux de participation sociale, surtout ceux que des aînés occupent majoritairement.

Moi, c'est plutôt une constatation. C'est qu'il faut dire que parmi les aînés normaux, ceux qui ne sont pas handicapés, il y a vraiment une répulsion face à nous autres. Les gens, aujourd'hui, sont ouverts. Parce qu'ils nous voient plus, ils nous rencontrent dans les centres d'achats, on travaille, on est sportifs, on est partout, on a une voiture, etc. Donc, ils sont plus habitués à nous voir. Mais les personnes âgées, les vieux, ils ne nous aiment pas. Pas du tout. Est-ce que ça vient parce qu'ils voient ce qu'ils pourraient être ? (Groupe de discussion)

Pour expliquer cette dynamique, les participants évoquent notamment «l'effet miroir » provoqué par leurs incapacités. Les aînés sans incapacités n'aimeraient pas voir, côtoyer, interagir avec des personnes handicapées, parce que ces dernières seraient évocatrices d'un inéluctable déclin à venir (Cohen, 1988). Les aînés sans incapacités seraient aussi plus enclins à ségréguer les personnes ayant des incapacités que les «plus jeunes", parce qu'ils auraient été socialisés dans une ère d'institutionnalisation, et donc moins habitués à fréquenter la diversité et la différence. La perception d'un risque d'exclusion ou de marginalisation constitue une menace au libre choix des personnes ayant des incapacités en matière de participation sociale, puisque ces dernières pourraient préférer l'évitement ou le retrait d'un milieu à la crainte d'un accueil mitigé. L'Illustration 2 est éloquente à ce sujet. On y raconte l'histoire vécue de Lise, qui préfère ne pas mentionner sa déficience auditive dans un nouveau milieu de bénévolat, car elle craint de s'attirer la pitié ou le rejet des autres membres du groupe. 
Illustration 2. Je bénévole, moi non plus
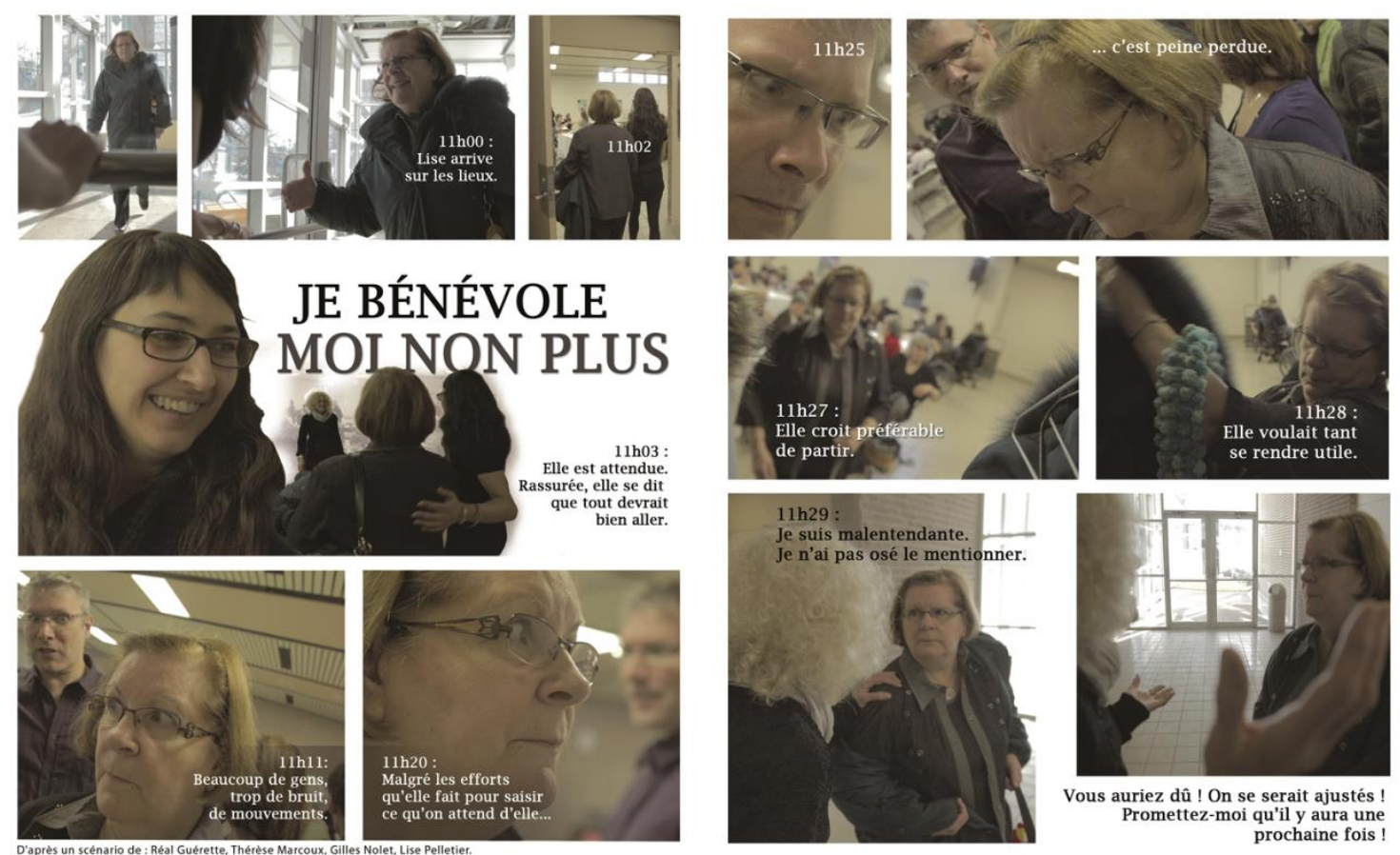

D'autres obstacles à la participation peuvent être environnementaux, rendant difficile l'accès physique aux espaces participatifs "généralistes » (c'est-à-dire hors des lieux « réservés 》 aux personnes handicapées). Face à des lieux non accessibles, c'est par le biais de pratiques personnelles d'autoacceptation, de négociation d'accommodements et de conquête de privilèges que les participants essaient tant bien que mal de s'incorporer. Cependant, en plus d'être énergivores, ces demandes sont susceptibles d'être mal reçues et peuvent même devenir humiliantes, renvoyant à des obstacles d'ordre relationnel. En même temps, ces efforts adaptatifs n'annihilent d'aucune manière le sens de la participation, qui reste possible et souhaitée même avec d'importantes exigences de soutien. En d'autres mots, les participants veulent que leurs possibilités de participation soient décentrées de leurs incapacités, d'un statut de personnes «spéciales ». D'ailleurs, pour plusieurs participants, l'expérience de vieillissement est gage d'aplanissement des différences en ce que de nombreux aînés développeront à leur tour des incapacités liées au vieillissement. En d'autres mots, pour des personnes ayant été perçues comme différentes toute leur vie, le vieillissement est perçu comme un moment potentiellement "égalisateur », puisque beaucoup d'aînés développent des incapacités. Voici quelques extraits des entretiens individuels menés permettant de saisir la complexité des pratiques en matière de vision et d'inclusion des aînés ayant des incapacités.

S'ils [les aînés handicapés visuels] pouvaient utiliser ces genres de jeux de cartes là [avec des chiffres agrandis ou des repères tactiles] facilement, du moins dans les activités de groupe, ils pourraient peut-être avoir le goût ou l'intérêt d'y aller. Là ils n'y vont pas parce qu'ils savent déjà en partant que ça va faire une montagne ou que ça va être un " tiraillage " pour l'acceptation de cette adaptation-là (Réal). 
Les gens [les autres membres du Club de collectionneurs] ont réalisé [mes besoins], il n'y avait jamais eu personne qui avait ces besoins-là. Mais là, la situation d'André qui est très impliqué et très actif méritait qu'on considère ça, tout le monde a été bien d'accord (André La).

[II faut] apprendre à ces gens-là [les personnes non handicapées] à côtoyer des gens qui vivent des différences et qu'on peut tous réussir à vivre ensemble si on y apporte certains accommodements ou certains ajustements (Réunion 2).

À quelque part aussi, il y a un petit peu de plaisir à vieillir, à retrouver le vieillissement. C'est un petit peu le "fun ", parce qu'enfin, on ressemble un petit peu à vous autres. II risque d'y avoir des personnes âgées qui sont en chaise roulante. Il y a du plaisir à ça. II y a un peu d'humour aussi. C'est amusant à quelque part. En tout cas, c'est comme une sorte de vengeance. Sympathique, je respecte. En même temps, on " fait plus partie de ", on est un petit peu moins à part (Groupe de discussion).

II ressort de ces résultats que si nous voulons garantir l'accès aux espaces participatifs sur les plans environnemental, symbolique et socioéconomique, il faut connaître et reconnaître la diversité des réalités et des besoins des aînés. Ceci demande de porter une attention inédite aux enjeux de l'altérité et de l'inter-culturalité, rompant avec des discours homogénéisant sur le vieillissement. Pourquoi ne pas faire en sorte que les milieux de participation donnent l'occasion de rencontrer l'Autre, avec ses différences et ses similitudes?

\section{DISCUSSION : POUR QUE LES CHOSES CHANGENT}

Le Projet Photoroman a permis de revisiter les discours dominants sur la participation sociale des aînés et de les transformer pour qu'ils puissent correspondre aussi aux situations de personnes vivant avec des incapacités, un processus de réécriture narrative bien mis en lumière par les étapes de la technique de l'incident critique (Stepney, 2006). Les douze participants ont d'abord exploré la trame de leurs pratiques de participation pour identifier les "moments critiques ", les occasions où le désir de s'insérer dans des milieux participatifs s'est soldé par l'incompréhension, le rejet, l'humiliation. Ces histoires, nombreuses, douloureuses, choquantes, ont ensuite été revues et analysées pour mieux comprendre comment la configuration contemporaine des discours sur le vieillissement, qui, se caractérisant par la généralisation et l'homogénéisation (Bytheway, Ward, Holland et Peace, 2007), génèrent des images d'un aîné participatif idéal en inhibant la diversité des situations de vieillissement (Baars, Dannefer, Phillipson et Walker, 2005). Lorsque la participation est conçue comme une métaphore de l'activité et de l'indépendance (Biggs, 2001, 2004; Minkler et Holstein, 2008), plusieurs questions critiques peuvent être formulées : tous les aînés souhaitent-ils participer tel qu'il est suggéré de le faire ? L'invitation qui leur est lancée est-elle limitée par les capacités personnelles ou des conditions de l'environnement physique, socioéconomique, culturelle? Des aînés pourraient-ils avoir envie ou besoin de façonner des modes participatifs "différents" des normes suggérées, mieux adaptés à leurs réalités?

Cet exercice d'analyse collective a mené à élaborer des scénarios esquissant une collection alternative d'images et de contenus, une plateforme de revendications quant à l'inclusion des aînés ayant des incapacités dans les lieux de participation à la vie collective. Autrement dit, le processus de création du photoroman a permis de préfigurer, de traduire en images et en mots les changements souhaités par des aînés ayant des incapacités quant à leur participation sociale, plus précisément : fréquenter les 
milieux « ordinaires » de participation; transformer leurs rapports avec les « autres aînés » dans une perspective interculturelle; être traité comme des citoyens; voir leur dignité respectée; et vivre des liens tissés de plaisir et de camaraderie dans leurs pratiques de participation sociale.

D'ailleurs, l'utilisation de la technique de l'incident critique pour analyser le processus et les résultats du Projet Photoroman révèle que la marginalisation expérimentée par les aînés ayant des incapacités dépasse l'esquisse de moments ponctuels, prenant plutôt la forme d'incidents répétés qui tissent un défaut quotidien d'inclusion sociale. Pour que cela change, il faut aussi que change notre vision collective de ce qu'est le fait de vivre avec des incapacités et de participer socialement, remplaçant une iconographie de l'anomalie par une iconographie de la diversité

En ce sens, le Projet Photoroman et ses résultats sont porteurs d'un potentiel de changement scientifique et social. Ils contribuent à combler d'importantes lacunes des écritsscientifiques concernant l'intersection vieillissement/incapacités (Jeppsson Grassman, Holme, Taghizadeh Larsson et Whitaker, 2012; Jönson et Taghizadeh Larsson, 2009; Kennedy, 2000; Minkler et Fadem, 2002; National Council on Ageing and Older People et National Disability Authority, 2006; Priestley, 2003; Putnam, 2002). Ils convient d'interroger des stéréotypes persistants dans les discours relatifs à la participation sociale des aînés (Lagacé, Laplante et Davignon, 2011; Rozanova, 2010), puisque l'attention presque hégémonique accordée aux perspectives de vieillissement « actif » et « en santé » masque les parcours et défis des personnes ne correspondant pas aux normes du «bien-vieillir », tout en ayant des implications pratiques par rapport à la manière dont les aînés ayant des incapacités sont perçus et accueillis dans les espaces collectifs de participation. Surtout, le photoroman Entre vous et nous balise un changement de pratiques d'intervention. Attachés au mouvement social des personnes ayant des incapacités et à ses persistantes, concrètes et efficaces revendications quant au respect des droits sociaux des personnes ayant des incapacités (Fougeyrollas, 2010), nos travaux visent avant tout à nourrir des réflexions et des approches en service social à la fois plus critiques et sensibles à la diversité des vieillissements.

RAYMOND, Émilie

GRENIER, Amanda

\section{RÉFÉRENCES}

Baars, J., D. Dannefer, C. Phillipson et A. Walker (2005). « Introduction: Critical perspectives in social gerontology », in J. Baars, D. Dannefer, C. Phillipson et A. Walker (dir.), Aging, globalization and inequality: the new critical gerontology, Amytiville (NY), Baywood, p. 1-14.

Balandin, S., G. Llewelly, A. Dew et L. Ballin (2006). "We couldn't function without volunteers": volunteering with a disability, the perspective of not-for-profit agencies ", International Journal of Rehabilitation Research, vol. 29, n², p. 131-136.

Bickenbach, J., C. Bigby, L. Salvador-Carulla, T. Heller, M. Leonardi, B. LeRoy, J. Mendez, M. Putnam et A. Spindel (2012). « The Toronto Declaration on Bridging Knowledge, Policy and Practice in Aging and Disability », International Journal of Integrated Care. 
Bigby, C., et S. Balandin (2005). «Another minority group: Use of aged care day programs and community leisure services by older people with lifelong disability », Australasian Journal on Ageing, vol. $24, n^{\circ} 1$, p. 14-18.

Biggs, S. (2001). « Toward critical narrativity: Stories of aging in contemporary social policy », Journal of Aging Studies, vol. 15, n 4, p. 303-316.

Biggs, S. (2004). « New agism: Age imperialism, personal experience and ageing policy », in S. O. Daatland et S. Biggs (dir.), Ageing and diversity: Multiple pathways and cultural migrations, Bristol, The Policy Press, p. 95-106.

Bradbury, H., et P. Reason (2003). « Action research: An opportunity for revitalizing research purpose and practices », Qualitative Social Work, vol. 2, n² 2, p. 155-175.

Brunelle, D., P. A. Harvey et S. Bédard (2005). « La nouvelle gestion publique en contexte », in D. Brunelle (dir.), Main basse sur l'État. Les partenariats public-privé au Québec et en Amérique du Nord, Montréal, Fides, p. 25-65.

Bytheway, B., R. Ward, C. Holland et S. Peace (2007). « The road to an age-inclusive society », in M. Bernard et T. Scharf (dir.), Critical Perspectives on Ageing Societies, Bristol, The Policy Press, p. 105-121.

Carrefour familial des personnes handicapées du Québec (2011). Actes du Forum sur la participation sociale des aînés handicapés. Vieillir avec un handicap, Québec, Carrefour familial des personnes handicapées.

Chevalier, J.M., et D.J. Buckles (2008). Social Analysis System (SAS2): A Guide to Collaborative Inquiry and Social Engagement, Thousand Oaks (CA), SAGE et International Development Research Centre.

Cohen, E.S. (1988). «The elderly mystique: Constraints on the autonomy of the elderly with disabilities », The Gerontologist, vol. 28, p. 24-31.

Côté, L., et B. Lévesque (2009). «L'État stratège, la citoyenneté active, la démocratie plurielle et la gouvernance partagée », in L. Côté, B. Lévesque et G. Morneau (dir.), État stratège et participation citoyenne, Québec, Presses de l'Université du Québec, p. 11-69.

Creswell, J. W. (2007). Qualitaitve inquiry and research design: Choosing among five approaches. Thousand Oaks (CA), SAGE.

De Buys, L., G.M. Boulton-Lewis, J.S. Tedman-Jones, H.E Edwards, M.F. Knox et C. Bigby (2008). "Issues of active ageing: Perceptions of older people with lifelong intellectual disability ", Australasian Journal on Ageing, vol. 27, $\mathrm{n}^{\circ} 2$, p. 67-71. 
Fals Borda, O. (2001). "Participatory (action) research in social theory: Origins and challenges », in P. Reason et $\mathrm{H}$. Bradbury (dir.), Handbook of action research: Participatory inquiry and practice, Thousand Oaks (CA), SAGE, p. 27-37.

Gouvernement du Québec (2005a). Loi assurant l'exercice des droits des personnes handicapées en vue de leur intégration sociale, scolaire et professionnelle.

Gouvernement du Québec (2005b). Une pleine participation des aînés au développement du Québec. Rapport de l'équipe de travail, Québec, Ministère de la Famille, des Aînés et de la Condition féminine.

Gouvernement du Québec (2007). Stratégie d'action en faveur des aînés. Un milieu de vie amélioré, une participation encouragée. Budget 2007-2008, Québec, Ministère des Finances.

Gouvernement du Québec (2009a). À part entière: pour un véritable exercice du droit à l'égalité. Politique sociale pour accroître la participation sociale des personnes handicapées, Québec, Office des personnes handicapées du Québec.

Gouvernement du Québec (2009b). Municipalité amie des aînés. Favoriser le vieillissement actif au Québec, Québec, Ministère de la Famille et des Aînés.

Gouvernement du Québec (2009c). Ministère de la Famille et des Aînés. Plan stratégique 2008-2012, Québec, Ministère de la Famille et des Aînés.

Gouvernement du Québec (2012a). Politique et plan d'action. Vieillir et vivre ensemble. Chez soi, dans sa communauté, au Québec. Québec, Ministère de la Famille et des Aînés.

Gouvernement du Québec (2012b). Qu'est-ce que le vieillissement actif? $<$ http://www.mfa.gouv.qc.ca/fr/aines/etre-actif/Pages/definition.aspx>, page consultée le 18 mai 2012.

Gouvernement du Québec (2013). Enquête québécoise sur les limitations d'activités, les maladies chroniques et le vieillissement 2010-2011. Utilisation des services de santé et des services sociaux des personnes avec incapacité, Québec, Institut de la statistique du Québec.

Hammersley, M., et P. Atkinson (2007). Ethnography: Principles in practice, New York (NY), Routledge.

Ingvaldsen, A. K., et S. Balandin (2011). " "If We are Going to Include Them We have to do it Before We Die": Norwegian Seniors' Views of Including Seniors with Intellectual Disability in Senior Centres. Journal of Applied Research in Intellectual Disabilities, vol. 24, n 6, p. 583-593.

Jeppsson Grassman, E., L. Holme, A, Taghizadeh Larsson et A. Whitaker (2012). " A long life with a particular signature: Life course and aging for people with disabilities ", Journal of Gerontological Social Work, vol. 55, n 2, p. 95-111.

Jeppsson Grassman, E., et A. Whitaker (2013). "Ageing with disability: An introduction », in E. Jeppson Grassman et A. Whitaker (dir.), Ageing with Disability: a lifecourse perspective, Bristol, Policy Press, p. 1-15. 
Jönson, H., et A. Taghizadeh Larsson (2009). « The exclusion of older people in disability activism and policies: A case of inadvertent ageism? », Journal of Aging Studies, vol. 23, n 1, p. 69-77.

Katz, S. (2005). Cultural aging: Life course, lifestyle, and senior worlds, Orchard Park (NY), Broadview Press.

Kennedy, J. (2000). "Responding to the disparities between disability research and aging research », Journal of Disability Policy Studies, vol. 11, n² 2, p. 120-123.

Lacroix, N., et É. Raymond (2014). La participation des aînés ayant des incapacités dans les milieux associatifs et communautaires: Des balises pour la réflexion et l'action. Québec, Université Laval, Chaire de recherche Marcelle-Mallet sur la culture philantropique.

Lagacé, M., J. Laplante et A. Davignon (2011). « Construction sociale du vieillir dans les médias écrits canadiens : de la lourdeur de la vulnérabilité à l'insoutenable légèreté de l'être », Communication \& Organisation, vol. 40, $\mathrm{n}^{\circ} 2$, p. 87-102.

Laliberté Rudman, D. (2006). "Shaping the active, autonomous and responsible modern retiree : An analysis of discursive technologies and their links with neo-liberal political rationality », Ageing \& Society, vol. 26, $\mathrm{n}^{\circ} 2$, p. 181-201.

Laliberté Rudman, D. (2011). "The duty to age well: Narrative negotiation of "Positive Aging" discourses", communication présentée au colloque "Theorizing Age : Challenging the Disciplines », Université de Maastricht, Pays-Bas, 6-9 octobre 2011.

Laliberté Rudman, D., S. Huot, L. Klinger, B.D. Leipert et M.M. Spafford (2010). « Struggling to maintain occupation while dealing with risk: The experiences of older adults with low vision », OTJR: Occupation, Participation and Health, vol. 30, no 2, p. 87-96.

Minkler, M., et P. Fadem (2002). " "Successful Aging" : A disability perspective », Journal of Disability Policy Studies, vol. 12, n 4, p. 229-235.

Minkler, M., et M.B. Holstein (2008). « From civil rights to... civic engagement? Concern of two critical gerontologists about a "new social movement" and what it portrends », Journal of Aging Studies, vol. 22, n², p. 196-204.

Morrow-Howell, N., J. Hinterlong et M. Sherraden (dir.) (2001). Productive aging: Concepts and challenges, Baltimore (MD), Johns Hopkins University Press.

Narushima, M. (2005). « Payback time: Community volunteering among older adults as a transformative mechanism », Ageing and Society, vol. 25, $n^{\circ} 4$, p. 567-584.

National Council on Ageing and Older People et National Disability Authority (2006). Ageing and disability: A discussion paper, Dublin, National Disability Authority.

Organisation des Nations unies (2002). Rapport de la deuxième Assemblée mondiale sur le vieillissement, New York (NY), Organisation des Nations unies. 
Organisation des Nations unies (2008). Guide to the National Implementation of the Madrid International Plan of Action on Ageing, New York (NY), Organisation des Nations Unies.

Organisation mondiale de la santé (2002). Vieillir en restant actif. Cadre d'orientation, Madrid, Organisation mondiale de la santé.

Organisation mondiale de la santé (2007). Guide mondial des villes-amies des aînés, Genève, Organisation mondiale de la santé.

Padgett, D. (2008). Qualitative Methods in Social Work Research, Thousand Oaks (CA), SAGE.

Pain, R., S. Kindon et M. Kesby (2007). "Participation action research: Making a difference to theory, practice and action ", in S. Kindon, R. Pain et M. Kesby (dir.), Participatory action Research approaches and methods: Connecting people, participation and place, New York (NY), Routledge, p. 26-32.

Priestley, M. (2003). Disability: A life course approach, Cambridge, Polity Press.

Putnam, M. (2002). « Linking aging theory and disability models: Increasing the potential to explore aging with physical impairment ", The Gerontologist, vol. 42, n 6, p. 799-806.

Raymond, É., D. Gagné, A. Sévigny et A. Tourigny (2008). La participation sociale des aînés dans une perspective de vieillissement en santé: Réflexion critique appuyée sur une analyse documentaire, Québec (QC), Institut national de santé publique du Québec, Agence de la santé et des services sociaux de la Capitale-Nationale, Centre d'excellence sur le vieillissement de Québec et Institut sur le vieillissement et la participation sociale des aînés de l'Université Laval.

Raymond, É., et A. Grenier (2012). "Vieillissement actif et aînés handicapés au Québec : Duo du possible ou mirage ? », Les politiques sociales, $n^{\circ \text { s } 1-2, ~ p . ~ 113-125 . ~}$

Raymond, É., et A. Grenier (2013). « Participation in policy discourse: New form of exclusion for seniors with disabilities? ", The Canadian Journal on Aging, vol. 32, n 2, p. 117-129.

Raymond, É., A. Grenier et J. Hanley (2014). "Community participation of older people with disabilities ", Journal of Community and Applied Social Psychology, vol. 24, n 1, p. 50-62.

Raymond, É., A. Grenier et N. Lacroix (2016). "La participation dans les politiques du vieillissement au Québec : discours de mise à l'écart pour les aînés ayant des incapacités ? ", Développement humain, handicap et changement social.

Raymond, É., et A. Pomerleau (2011). «Le forum du 4 mai 2010 : Des résultats de recherche qui font du chemin ", in A. Pomerleau (dir.), Vieillir avec un handicap. Actes du Forum sur la participation sociale des aînés, Québec QC), Carrefour familial des personnes handicapées, p. 31-47.

Raymond, É., A. Sévigny et A. Tourigny (2011). "La participation sociale : ce qu'en disent des aînés handicapés «, in A. Pomerleau (dir.), Vieillir avec un handicap. Actes du Forum sur la participation sociale des aînés, Québec (QC), Carrefour familial des personnes handicapées, p. 9-29. 
Raymond, É., A. Sévigny et A. Tourigny (2012). Participation sociale des aînés : La parole aux aînés et aux intervenants, Québec (QC), Institut national de santé publique du Québec, Institut sur le vieillissement et la participation sociale des aînés de l'Université Laval, Direction de santé publique de l'Agence de la santé et des services sociaux de la Capitale-Nationale, Centre d'excellence sur le vieillissement de Québec du Centre hospitalier affilié universitaire de Québec.

Reason, P., et H. Bradbury (dir.) (2008). The SAGE Handbook of Action Research: Participative Inquiry and Practice, Thousand Oaks (CA), SAGE.

Rowe, J., et R. Kahn (1998). Successful Aging, New York (NY), Dell Publishing.

Rozanova, J. (2010). "Discourse of successful aging in The Globe \& Mail: Insights from critical gerontology », Journal of Aging Studies, vol. 24, n 4, p. 213-222.

Savard, J., N. Leduc, P. Lebel, F. Béland et H. Bergman (2009). « Determinants of Adult Day Center Attendance Among Older Adults With Functional Limitations », Journal of Aging And Health, vol. 21, $\mathrm{n}^{\circ} 7$, p. 985-1015.

Statistique Canada (2009). Canadiens dévoués, Canadiens engagés : Points saillants de l'Enquête canadienne de 2007 sur le don, le bénévolat et la participation, Ottawa (ON), Gouvernement du Canada.

Statistique Canada (2012). Canadiens dévoués, Canadiens engagés : rapport de tableaux, Ottawa (ON), Gouvernement du Canada.

Stepney, P. (2006). « Mission Impossible? Critical practice in social work », British Journal of Social Work, vol. 36, n 8 , p. 1289-1307.

Tang, F. (2009). «Late-life volunteering and trajectories of physical health », Journal of Applied Gerontology, vol. 28, n 4, p. 524-533.

United Nations Organization (2005). The framework for monitoring, review and appraisal of the Madrid International Plan of Action on Ageing, New York (NY), United Nations.

United Nations Organization (2014). Enable: Priority themes. http://www.un.org/disabilities/default.asp?id=33. page consultée le 13 février 2014. 